\title{
Power-Efficient Non-coherent Space-Time Constellations
}

\author{
Mohammad Jaber Borran, Ashutosh Sabharwal, and Behnaam Aazhang \\ ECE Department, MS-366, Rice University, Houston, TX 77005-1892 \\ Email: $\{$ mohammad,ashu,aaz $\} @$ rice.edu
}

\begin{abstract}
We consider the problem of digital communication in a Rayleigh flat-fading environment using a multiple-antenna system, when the channel state information is available neither at the transmitter nor at the receiver. It is known that at high SNR, or when the coherence interval is much larger than the number of transmit antennas, a constellation of unitary matrices can achieve the capacity of the non-coherent system. However, at low SNR, high spectral efficiencies, or for small values of coherence interval, the unitary constellations lose their optimality and fail to provide an acceptable performance. In an earlier work, using a design criterion based on the KL distance between distributions, we have shown, for the case of a single transmit antenna, that at low SNR, a multilevel constellation can provide better error rate performance than the unitary constellation of the same size. In this work, we extend those single antenna results to the case of multiple transmit antennas. The interesting result of this work is that the same idea of multilevel constellations can be generalized to design low-power non-coherent constellations for multiple antennas. The resulting constellations overlap with the unitary constellations at high SNR, but at low SNR they have a multilevel structure and show significant performance improvement over unitary constellations of the same size. The performance improvement becomes especially more significant when a large number of receive antennas are used. This property, together with the facts that the proposed constellations eliminate the need for training sequences and are most suitable for low SNR, makes them a good candidate for uplink communication in wireless systems.
\end{abstract}

\section{Index Terms}

Non-coherent constellations, space-time codes, multiple antenna systems, fading channels, channel coding, wireless communications.

\section{INTRODUCTION}

In wireless communication systems, channel state information at the receiver is usually obtained through transmission of periodic training symbols. For training to be effective, it is assumed that the channel remains almost constant for a period which is referred to as coherence interval. The transmitter uses some part of the coherence interval to transmit training symbols, and the rest of it for actual data communication. The receiver, estimates the channel using the knowledge of transmitted symbols in training period, and then uses that estimate for detection of the data symbols (coherent detection). For a slowly fading channel, the channel parameters remain almost constant for a relatively long time, resulting in large coherence intervals. Channel training is needed only once during one coherence interval, therefore for slowly fading channels the training overhead is small. As the rate of channel variations increases, the coherence interval becomes shorter and the training overhead increases, making coherent detection less appealing. In fast fading channels, the situation is even worse, because the coherence interval is so short that the fading coefficients can change into new values before being learned by the receiver through training signals. A non-coherent scheme, where receiver detects the transmitted symbols without having any information about the current realization of the channel, is more suitable for these scenarios. Another application of non-coherent constellations can be in IEEE 
802.11 systems for transmission of short control packets (such as RTS and CTS), where training sequences have a large overhead in terms of the fraction of time and power spent on them.

It is known [1,2] that at high SNR, or when the coherence interval, $T$, is much larger than the number of transmit antennas, $M$, capacity can be achieved by using a constellation of unitary matrices (i.e., with orthonormal columns). However, at low SNR or for small values of $T$, the unitary constellations lose their optimality especially at high spectral efficiencies. In [3], we have shown that a design criterion based on maximizing the minimum Kullback-Leibler (KL) distance between the conditional distributions, can result in a significant performance improvement for single transmit antenna non-coherent constellations at low SNR. The resulting signal sets are multilevel constellations of several $T$-dimensional concentric hemispheres, and provide a larger minimum KL distance compared to the unitary constellation of the same size.

In this work, we extend the single antenna results of [3] to the case of multiple transmit antennas. We show that the idea of multilevel constellations can be extended to the case of multiple transmit antennas by using unitary space-time constellations at each level. The only difference is that the angular distance between constellation vectors in the single-antenna case should be replaced with the more general metric of chordal distance between subspaces in the multiple-antenna case. Similar to the single-antenna case, at high SNR's or low rates, the KL-based design criterion reduces to the design criterion for unitary constellations, confirming their high SNR optimality. However, at low SNR, the new design criterion results in multilevel constellations, which show significant performance improvement over the unitary constellations of the same size, especially when a large number of receive antennas are used.

The rest of this paper is organized as follows. In Section II, we introduce the system model. In Section III, we derive the design criterion for multiple-antenna constellations, and propose a multilevel construction with unitary constellations at each level. In Section IV, we present our simulation results and show that multilevel constellations can provide significant performance improvement over unitary constellations of the same size. Finally, in Section V we bring some concluding remarks.

\section{System MODEL}

We consider a communication system with $M$ transmit and $N$ receive antennas in a block Rayleigh flat fading channel with coherence interval of $T$ symbol periods (i.e., we assume that the fading coefficients remain constant during blocks of $T$ consecutive symbol intervals, and change to new, independent values at the end of each block). We use the following complex baseband notation

$$
X=S H+W
$$

where $S$ is the $T \times M$ matrix of transmitted signals, $X$ is the $T \times N$ matrix of received signals, $H$ is the $M \times N$ matrix of fading coefficients, and $W$ is the $T \times N$ matrix of the additive received noise. Elements of $H$ and $W$ are assumed to be statistically independent, identically distributed circular complex Gaussian

random variables from the distribution $\mathcal{C N}(0,1)$. We use the power constraint $\sum_{t=1}^{T} \sum_{m=1}^{M} \mathbb{E}\left\{\left|s_{t m}\right|^{2}\right\}=$ $T P$, where $s_{t m}$ 's are the elements of the signal matrix $S$.

With the above assumptions, the conditional probability density of the received signal can be written as

$$
p(X \mid S)=\mathbb{E}_{H}\{p(X \mid S, H)\}=\frac{\exp \left\{-\operatorname{tr}\left[\left(I_{T}+S S^{H}\right)^{-1} X X^{H}\right]\right\}}{\pi^{T N} \operatorname{det}^{N}\left(I_{T}+S S^{H}\right)} .
$$

Having observed the received matrix $X$, the Maximum Likelihood (ML) detector chooses the constellation point $S$, as the detected symbol, which maximizes the above expression. Our goal is to design matrix constellations which achieve the smallest average error probability with this ML detector. However, similar to other code and constellation design problems, it is more convenient to work with the worst case scenario, and 
minimize the maximum pairwise error probability, instead of the average error probability. Unfortunately, the exact expression or even the Chernoff upper bound for the pairwise error probability does not seem to be tractable for a general non-coherent constellation. Moreover, except for the special case of unitary constellations, the pairwise error probabilities are not symmetric. Therefore, inspired by the Stein's lemma, in [3] we have proposed the use of Kullback-Leibler (KL) distance between conditional distributions as design criterion. In [4], we have shown that, even though the KL distance does not serve as an error exponent for the ML detector, the pairwise error probability decreases as the KL distance increases. Therefore, the KL distance can be used as a valid constellation design criterion for the ML detector.

In [3], the KL distance between $p_{i}=p\left(X \mid S_{i}\right)$ and $p_{j}=p\left(X \mid S_{j}\right)$ has been derived as

$$
\mathcal{D}\left(p_{i} \| p_{j}\right)=N \operatorname{tr}\left\{\left(I_{T}+S_{i} S_{i}^{H}\right)\left(I_{T}+S_{j} S_{j}^{H}\right)^{-1}\right\}-N \ln \operatorname{det}\left\{\left(I_{T}+S_{i} S_{i}^{H}\right)\left(I_{T}+S_{j} S_{j}^{H}\right)^{-1}\right\}-N T .
$$

For the case of a single transmit antenna, the above expression reduces to

$$
\mathcal{D}\left(p_{i} \| p_{j}\right)=\underbrace{\frac{1+\left\|S_{i}\right\|^{2}}{1+\left\|S_{j}\right\|^{2}}-\ln \left(\frac{1+\left\|S_{i}\right\|^{2}}{1+\left\|S_{j}\right\|^{2}}\right)-1}_{\mathcal{D}_{1}\left(p_{i} \| p_{j}\right)}+\underbrace{\frac{\left\|S_{i}\right\|^{2}\left\|S_{j}\right\|^{2} \sin ^{2}\left(\angle S_{i}, S_{j}\right)}{1+\left\|S_{j}\right\|^{2}}}_{\frac{\left\|S_{i}\right\|^{2}}{\left\|S_{j}\right\|^{2}} \mathcal{D}_{2}\left(p_{i} \| p_{j}\right)},
$$

where $\mathcal{D}_{1}$ denotes the distance between two points which lie on a straight line passing through the origin, and $\mathcal{D}_{2}$ denotes the distance between two points lying on a $T$-dimensional sphere. The above partitioning of the KL distance was used in [3] to suggest the use of a multilevel constellation for this case.

\section{Design Criterion for MultiPle-Antenna Non-COHEREnt Constellations}

In [1], it has been shown that the capacity of non-coherent systems can be achieved by an orthogonal random signal matrix constructed as the product $S=\Phi V$, where $\Phi$ is an isotropically distributed $T \times M$ matrix with orthonormal columns, and $V$ is an independent $M \times M$ real, non-negative, diagonal matrix. Therefore, in designing matrix constellations for multiple-antenna non-coherent systems, we will confine ourselves to constellations of orthogonal matrices (i.e., matrices with orthogonal columns). With this assumption, the KL distance expression in (3) can be written as

$$
\mathcal{D}\left(p_{i} \| p_{j}\right)=\sum_{m=1}^{M}\left\{\frac{1+\left\|S_{i m}\right\|^{2}}{1+\left\|S_{j m}\right\|^{2}}-\ln \left(\frac{1+\left\|S_{i m}\right\|^{2}}{1+\left\|S_{j m}\right\|^{2}}\right)-1+\frac{\left\|S_{i m}\right\|^{2}\left\|S_{j m}\right\|^{2}-\sum_{k=1}^{M}\left|S_{i k} \cdot S_{j m}\right|^{2}}{1+\left\|S_{j m}\right\|^{2}}\right\},
$$

where we have used the notation $S_{l m}$ to denote the $m$ th column of $S_{l}$. To further simplify the KL expression and the design problem, we impose other constraints on the constellation points, as explained in the following subsections.

\section{A. $S_{i k} \cdot S_{j m}=0$ for all $i, j$, and $k \neq m$}

In this case, each column of any constellation matrix is orthogonal to all of the other columns of all of the constellation points. If we define the $m$ th column space of the constellation as the linear space spanned by the $m$ th column of all of the constellation matrices, this constraint means that any two different column spaces of the constellation are orthogonal. With this assumption, the KL distance expression in (5) reduces to

$$
\mathcal{D}\left(p_{i} \| p_{j}\right)=\sum_{m=1}^{M}\left\{\frac{1+\left\|S_{i m}\right\|^{2}}{1+\left\|S_{j m}\right\|^{2}}-\ln \left(\frac{1+\left\|S_{i m}\right\|^{2}}{1+\left\|S_{j m}\right\|^{2}}\right)-1+\frac{\left\|S_{i m}\right\|^{2}\left\|S_{j m}\right\|^{2} \sin ^{2}\left(\angle S_{i m}, S_{j m}\right)}{1+\left\|S_{j m}\right\|^{2}}\right\} .
$$


As we see, the KL distance in this case breaks into summation of $M$ KL distances of the form (4), and each term depends only on one column from each matrix. This means that we can partition the $T$-dimensional space as direct summation of $M$ orthogonal subspaces (which will serve as column spaces of the constellation), and use the method of [3] to independently construct single-antenna constellations inside each subspace. The multiple-antenna constellation then can be obtained by forming the Cartesian product of all of these single-antenna constellations. An example of this approach, is the multiple-antenna extension of the PSK-type constellations of [5]. Assuming that $T=2 M$, in [5] the $T$-dimensional space is suggested to be partitioned into $M$ 2-dimensional subspaces, each one generated by a different pair of elements of the standard basis. Inside each subspace (or equivalently, for each transmit antenna over two time instants), a single-antenna PSK-type constellation is used. In [5], instead of considering the Cartesian product of the single-antenna constellations, the same constellation point is repeated on different antennas to achieve the maximum available diversity. However, considering the fact that in this case, $T$ is $M$ times larger than the single-antenna case, this results in a decrease in rate by a factor of $M$. Using the Cartesian product maintains the same rate as the single-antenna case, however it does not provide any performance improvement. The minimum KL distance of the resulting multiple-antenna constellation is the same as the minimum KL distance of the single-antenna constituent constellation. In fact, this approach is equivalent to designing a single-antenna constellation for $T=2$, and forming uncoded blocks of length $T=2 M$ and transmitting them over the same antenna.

B. $S_{l}^{H} S_{l}=d_{l} I_{M}$ for $l=1, \cdots, L$

The assumption in this case is that each signal matrix is a scalar multiple of a unitary matrix. With this assumption, the KL distance in (3) reduces to

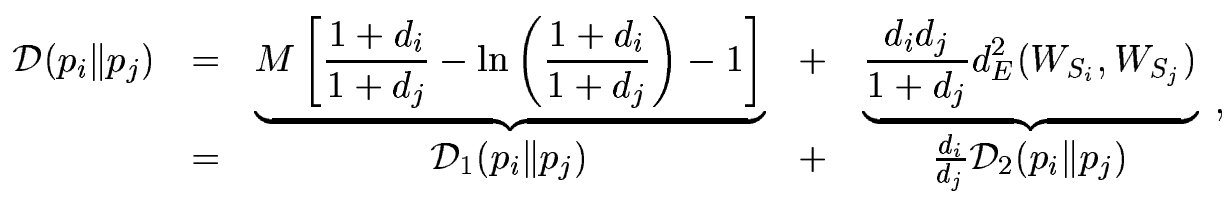

where $d_{E}^{2}\left(W_{S_{i}}, W_{S_{j}}\right)$ is the square Euclidean distance [5] or chordal distance [6] between the two subspaces $W_{S_{i}}$ and $W_{S_{j}}$ spanned by columns of $S_{i}$ and $S_{j}$, defined as

$$
d_{E}^{2}\left(W_{S_{i}}, W_{S_{j}}\right)=\sum_{m=1}^{M} d_{E}^{2}\left(\frac{S_{i m}}{\sqrt{d_{i}}}, W_{S_{j}}\right)=\sum_{m=1}^{M}\left\{\frac{\left\|S_{i m}\right\|^{2}}{d_{i}}-\sum_{k=1}^{M} \frac{\left|S_{i m} \cdot S_{j k}\right|^{2}}{d_{i} d_{j}}\right\},
$$

$\mathcal{D}_{1}$ denotes the distance between two constellation points which represent the same $M$-dimensional subspace of the $T$-dimensional space, and $\mathcal{D}_{2}$ denotes the distance between two constellation points with the same power which represent two different $M$-dimensional subspaces. In general, the overall distance is greater than or equal to either of these parts. Recalling that the unitary constellations are designed to maximize the Euclidean distance between subspaces, the above partitioning of the KL distance suggests partitioning the signal space into subsets of unitary constellations, $C_{1}, \cdots, C_{K}$, with columns of square norm $\rho_{1}, \cdots, \rho_{K}$, containing $l_{1}, \cdots, l_{K}$ points, respectively. With the above partitioning, we define the intra-subset and inter-subset distances as

$$
\mathcal{D}_{\text {intra }}(k)=\min _{S_{i}, S_{j} \in C_{k}} \frac{\rho_{k}^{2}}{1+\rho_{k}} d_{E}^{2}\left(S_{i}, S_{j}\right),
$$

and

$$
\mathcal{D}_{\text {inter }}\left(k, k^{\prime}\right)=M\left[\frac{1+\rho_{k}}{1+\rho_{k^{\prime}}}-\ln \left(\frac{1+\rho_{k}}{1+\rho_{k^{\prime}}}\right)-1\right]
$$


Without loss of generality, we can assume that $\rho_{1}<\rho_{2}<\cdots<\rho_{K}$, and solve the simplified maximin problem

$$
\begin{gathered}
\operatorname{maximize} \\
1 \leq K \leq L, \frac{M}{L} \sum_{k=1}^{K} l_{k} \rho_{k}=T P, \sum_{k=1}^{K} l_{k}=L \\
0 \leq \rho_{1}<\rho_{2}<\cdots<\rho_{K}
\end{gathered}
$$

to find the optimal $L$-point multilevel unitary constellation of $T \times M$ matrices with average power $P$. At each level, we can use any existing unitary construction and substitute, for $\mathcal{D}_{\text {intra }}(k)$ in $(9)$, the best achievable KL distance with that construction and size $l_{k}$.

In (11), $K$ and $l_{1}, \cdots, l_{K}$ are discrete variables, while $\rho_{1}, \cdots, \rho_{K}$ are continuous variables. For any fixed value of $K$ and $l_{1}, \cdots, l_{K}$ satisfying the specified constraints, (11) reduces to a continuous optimization over $\rho_{1}, \cdots, \rho_{K}$, which can be solved numerically. Even though in (11) we have allowed $K$ to range from 1 to $L$, in practice we do not need to try all of these possible values for $K$. Starting from $K=1$, and increasing the value of $K$ by one each time, we can stop the search once the optimum minimum distance of the solution stops increasing. Moreover, since the intra-subset distance is an increasing function of $\rho_{k}$, it can be shown that the optimum constellation also satisfies the extra constraint $l_{1} \leq l_{2} \leq \cdots \leq l_{K-1}$, which can be used to further restrict the domain of our search.

From (7), we also observe that at high SNR, $\mathcal{D}_{1}$ becomes a constant, or its minimum grows at most logarithmically with SNR (when $d_{i}=0$ ), whereas $\mathcal{D}_{2}$ grows linearly with SNR for non-zero constellation points from different subspaces. As a result, at high $\mathrm{SNR}, \mathcal{D}_{2}$ becomes the dominant term, and the KL-based design criterion reduces to the Euclidean-based design criterion of the unitary constellations, confirming the high SNR optimality of the unitary constellations.

\section{Simulation Results}

In this section, we present the results of our simulations to evaluate the performance of the proposed constellations and compare them with the existing results. Figures 1(a) and 1(b) show the error rate performance of the proposed constellations. In these examples, we have used the systematic unitary designs of [7] as the constituent subsets of the multilevel constellations. The comparisons are between these systematic constellations and their multilevel versions. Figure 1(a) shows the block error rate performance of the 16 and 32-point two-antenna constellations with $T=3$ and $T=4$ (resulting in spectral efficiencies of 1.33 and $1.25 \mathrm{~b} / \mathrm{s} / \mathrm{Hz}$ ), respectively. The horizontal axis is the number of receive antennas, with SNR kept fixed at $0 \mathrm{~dB}$. As we see, the multilevel constellations can save up to 4 receive antennas at SNR's as low as $0 \mathrm{~dB}$. In [3], we have shown even larger savings for the case of single transmit antenna with $T=2$.

Figure 1(b) compares the performances of 16-point, one and two transmit antenna constellations for $T=2$ and $T=3$ (resulting in spectral efficiencies of 2 and $1.33 \mathrm{~b} / \mathrm{s} / \mathrm{Hz}$ ), respectively. The horizontal axis is SNR, and the receiver is assumed to have 10 receive antennas. Even if multiple receive antennas are not available, similar results can be obtained by encoding across several fading blocks using an outer code. For each point in the curves corresponding to the multilevel constellations in Figure 1(b), a separate optimization problem with appropriate power constraint has been solved and the resulting constellation has been used to evaluate the performance. We observe that the multilevel unitary constellation can provide up to $3 \mathrm{~dB}$ gain over its corresponding one-level unitary constellation at low SNR. We also notice that as SNR increases, the two curves become closer, which is expected, recalling the optimality of the unitary constellations at high SNR. 


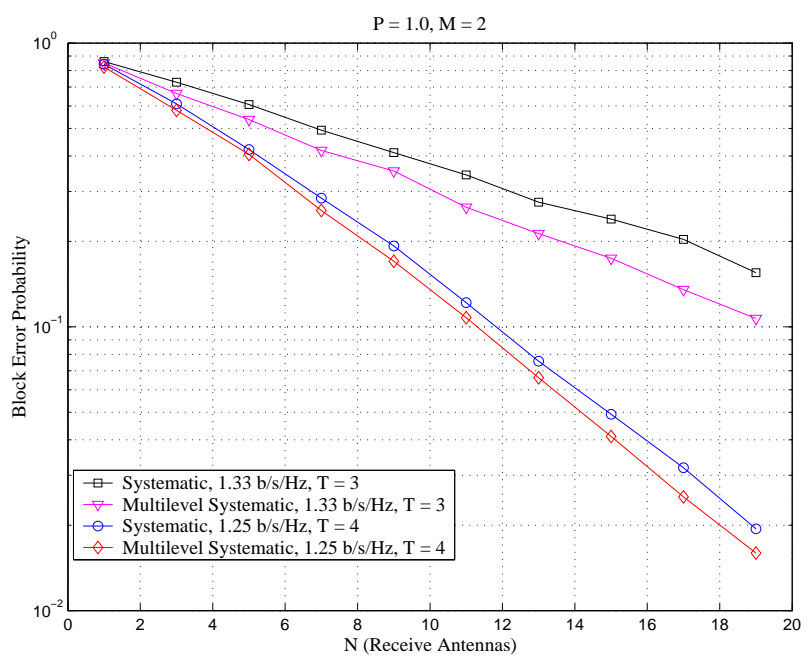

(a)

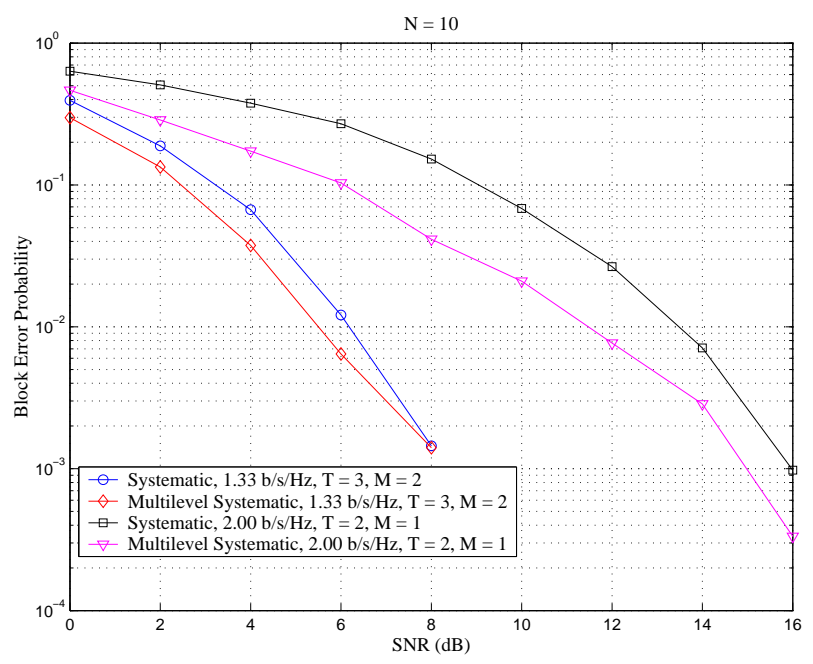

(b)

Fig. 1. Performance comparison of one and two-antenna systematic constellations of [6] and their multilevel versions vs. (a) number of receive antennas, and (b) SNR

\section{Conclusions}

We considered the problem of non-coherent communication over a block Rayleigh flat fading channel. We derived the design criterion for multiple-antenna constellations based on the Kullback-Leibler distance between conditional distributions. We presented a multilevel construction with unitary constellations at each level. Any existing unitary construction can be used inside the levels, and the number of levels, the number of points at each level, and the radiuses of different levels are obtained by solving a maximin problem. At high SNR, the multilevel construction reduces to the unitary constellations, confirming their high SNR optimality. Our simulations show that at low SNR or high spectral efficiencies, multilevel constellations can provide significant performance improvement over unitary constellations of the same size.

\section{REFERENCES}

[1] T. L. Marzetta and B. M. Hochwald, "Capacity of a mobile multiple-antenna communication link in Rayleigh flat fading," IEEE Transactions on Information Theory, vol. 45, no. 1, pp. 139-157, Jan. 1999.

[2] B. M. Hochwald and T. L. Marzetta, "Unitary space-time modulation for multiple-antenna communications in Rayleigh flat fading," IEEE Transactions on Information Theory, vol. 46, no. 2, pp. 543-564, March 2000.

[3] M. J. Borran, A. Sabharwal, B. Aazhang, and D. H. Johnson, "On design criteria and construction of non-coherent space-time constellations," in Proceedings of the IEEE International Symposium on Information Theory, July 2002.

[4] M. J. Borran, A. Sabharwal, and B. Aazhang, "On design criteria and construction of non-coherent space-time constellations," to be sumbitted to the IEEE Transactions on Information Theory, Special Issue on Space-Time Transmission, Reception, Coding, and Signal Design, Oct. 2002.

[5] V. Tarokh, "On the design of the first unitary space-time codes that have simple encoding/decoding algorithm," in Proceedings of the 39th Annual Allerton Conference on Communications, Control and Computing, Monticello, IL, Oct. 2001.

[6] J. H. Conway, R. H. Hardin, and N. J. A. Sloane, "Packing lines, planes, etc: packings in Grassmannian space," Experimental Math, vol. 5, pp. 139-159, 1996.

[7] B. M. Hochwald et al, "Systematic design of unitary space-time constellations," IEEE Transactions on Information Theory, vol. 46, no. 6, pp. 1962-1973, Sept. 2000. 